\title{
AIDS e infecção pelo HIV no Brasil: uma epidemia multifacetada
}

\author{
AIDS and HIV infection in Brazil: a multifaceted epidemic
}

Ana Maria de Brito', Euclides Ayres de Castilho²e Célia Landmann Szwarcwald ${ }^{3}$

\begin{abstract}
Resumo A epidemia da infecção pelo HIV e da AIDS constitui fenômeno global, dinâmico e instável, traduzindose por verdadeiro mosaico de sub-epidemias regionais. Resultante das profundas desigualdades da sociedade brasileira, a propagação da infecção pelo HIV e da AIDS revela epidemia de múltiplas dimensões que vem sofrendo transformações epidemiológicas significativas. Inicialmente restrita aos grandes centros urbanos e marcadamente masculina, a atual epidemia do HIV e da AIDS caracteriza-se pelos processos de heterossexualização, feminização, interiorização e pauperização. As mudanças no perfil da AIDS no Brasil devem-se à difusão geográfica da doença a partir dos grandes centros urbanos em direção aos municípios de médio e pequeno porte, ao aumento da transmissão por via heterossexual e ao persistente crescimento dos casos entre usuários de drogas injetáveis. O aumento da transmissão por contato heterossexual implica no crescimento substancial de casos em mulheres, o qual tem sido apontado como uma das mais importantes características do atual quadro da epidemia no Brasil.
\end{abstract}

Palavras-chaves: HIV. AIDS. Epidemiologia. Brasil.

Abstract The HIVIAIDS epidemic is a dynamic unstable global phenomenon, constituting a veritable mosaic of regional sub-epidemics. As a consequence of the deep inequalities that exist in Brazilian society, the spread of HIV infection has revealed an epidemic of multiple dimensions undergoing extensive epidemiological transformations. Initially restricted to large urban centers and markedly masculine, the HIV/AIDS epidemic is currently characterized by heterosexualization, feminization, interiorization and pauperization. The evolution of the profile of AIDS in Brazil is above all due to the geographical diffusion of the disease from large urban centers towards medium and small municipalities in the interior, to the increase in heterosexual transmission and the persistent growth of cases among injecting drug users. The increase in transmission through heterosexual contact has resulted in substantial growth of cases among women, which has been pointed out as the most important characteristic of the epidemic's current dynamic in Brazil.

Key-words: HIV. AIDS. Epidemiology. Brazil.

A identificação, em 1981, da síndrome da imunodeficiência adquirida, habitualmente conhecida como AIDS, tornou-se um marco na história da humanidade. A epidemia da infecção pelo vírus da imunodeficiência humana (HIV) e da AIDS representa fenômeno global, dinâmico e instável, cuja forma de ocorrência nas diferentes regiões do mundo depende, entre outros determinantes, do comportamento humano individual e coletivo. A AIDS destaca-se entre as enfermidades infecciosas emergentes pela grande magnitude e extensão dos danos causados às populações e, desde a sua origem, cada uma de suas características e repercussões tem sido exaustivamente discutida pela comunidade científica e pela sociedade em geral.

A epidemia de AIDS mostra-se bastante complexa e configura-se como verdadeiro mosaico de subepidemias regionais. Na tentativa de descrever as principais características da dinâmica epidemiológica da AIDS no mundo, várias tipologias têm sido propostas $^{5}$. A mais recente, e que melhor traduz o atual momento da epidemia, define três tipos ${ }^{18}$ : epidemia nascente (ou baixo nível) - corresponde a países onde a prevalência da infecção pelo HIV mostra-se menor do que $5 \%$ em todas as subpopulações com comportamento de alto risco à exposição ao vírus; epidemia concentrada - referente a países onde a prevalência da infecção pelo HIV é superior a $5 \%$ em uma ou mais subpopulações com comportamento de alto risco, mas a prevalência entre gestantes atendidas em clínicas de pré-natal revela-se menor do que $5 \%$, e epidemia generalizada - ocorrendo nos países onde a infecção pelo HIV deixou de ser restrita as subpopulações de comportamento de risco, que

\footnotetext{
1. Departamento de Medicina Social da Faculdade de Ciências Médicas da Universidade de Pernambuco, Recife, PE. 2. Departamento de Medicina Preventiva da Faculdade Medicina, Universidade de São Paulo, SP. 3. Departamento de Informações para a Saúde, Centro de Informações Científicas e Tecnológicas/FIOCRUZ

Endereço para correspondência: Dr. Euclides Ayres de Castilho. Faculdade de Medicina/USP. Av. Dr. Arnaldo 455, 01246-903 São Paulo, SP. e-mail: castilho@usp.br

Recebido para publicação em 23/2/2001.
} 
apresentam elevadas taxas de prevalência da infecção, e a prevalência entre gestantes atendidas em clínicas de pré-natal mostrou-se igual ou superior a $5 \%$. A epidemia no Brasil seria do tipo concentrada, juntamente com alguns países da América do Sul, Sudão, Tailândia e Etiópia. Por outro lado, a epidemia generalizada ocorre em países da África subsaariana e Haiti, enquanto observou-se tipo baixo nível em países como a Argélia, Chile, Costa Rica, Cuba, Suriname, Federação Russa e Polônia.

Como resultado das profundas desigualdades da sociedade brasileira, a propagação da infecção pelo HIV no País revela epidemia de múltiplas dimensões que vem, ao longo do tempo, sofrendo transformações significativas em seu perfil epidemiológico. De epidemia inicialmente restrita a alguns círculos cosmopolitas das denominadas metrópoles nacionais - São Paulo e Rio de Janeiro - e marcadamente masculina, que atingia prioritariamente homens com prática sexual homossexual e indivíduos hemofílicos, depara-se, hoje, com quadro marcado pelos processos da heterossexualização, da feminização, da interiorização e da pauperização. 0 aumento da transmissão por contato heterossexual resulta em crescimento substancial de casos em mulheres, o que vem sendo apontado como o mais importante fenômeno para o atual momento da epidemia. Descrever-se-á, neste artigo, as principais características das mudanças ocorridas no perfil da infecção pelo HIV e da AIDS no Brasil.

\section{TENDÊNCIA ESPAÇO-TEMPORAL DA AIDS: A INTERIORIZAÇÃO}

A partir do eixo Rio-São Paulo, os casos de AIDS disseminaram-se para as demais regiões, inicialmente às metrópoles regionais, a partir do final da década de oitenta. As transformações no perfil da AIDS no Brasil,

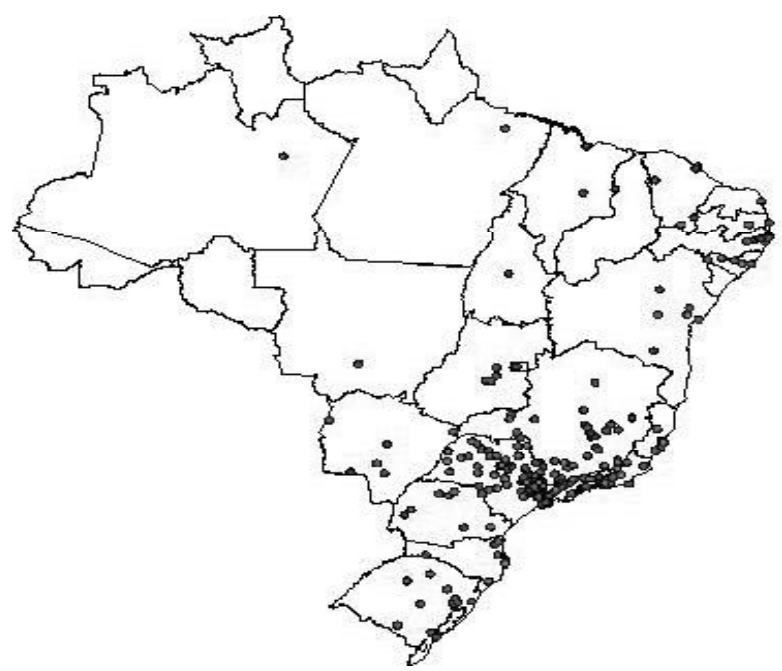

Figura 1 - Distribuição espacial dos municípios com pelo menos um caso de AIDS registrado, no Brasil, no período de 1980 a 1996 - Fonte: CN-DST/AIDS/SPS - Ministério da Saúde, 2000. embora com dinâmicas regionais e populacionais distintas devem-se, sobretudo, a difusão geográfica da doença a partir dos grandes centros urbanos em direção aos municípios de médio e pequeno porte do interior do País, ao aumento da transmissão heterossexual e ao persistente crescimento dos casos entre usuários de drogas injetáveis (UDI). Atualmente, a epidemia não mais se restringe aos grandes centros urbanos e já atinge $59 \%$ dos 5.507 municípios brasileiros. Os cartogramas representados nas Figuras 1, 2 e 3 ilustram a progressiva expansão da epidemia do litoral sudeste para as regiões Nordeste, CentroOeste e Norte, considerando-se a distribuição espacial dos municípios com pelo menos um caso de AIDS registrado, para os períodos de 1980 a 1986, de 1987 a 1993 e de 1994 a $2000^{12}$.

Desde o início da epidemia, em 1980, até junho de 2000 foram notificados à Coordenação Nacional de DST e AIDS do Ministério da Saúde, 190.949 casos de AIDS. Desses casos, 6.750 são crianças, 139.502 adultos masculinos e 44.697 adultos femininos. Com registro de ocorrência de casos em quase todo o território nacional, a distribuição da AIDS, no entanto, não mostrase homogênea quanto às regiões de residência, sexo, idade, grau de escolaridade nem no que se refere às categorias de transmissão. Observando-se maior concentração de casos nas regiões Sudeste e Sul, as taxas de incidência nos últimos anos evoluíram, em todo o País, de 8 por 100 mil habitantes, em 1991, para 13,7 por 100 mil habitantes, em 1998 (Figura 4) e apresenta grande variação entre os diferentes estados da Federação ${ }^{12}$.

Em estudo recente, Szwarcwald et al ${ }^{15}$ caracterizaram a evolução temporal da epidemia da AIDS no Brasil. Para o conjunto do País, e considerando

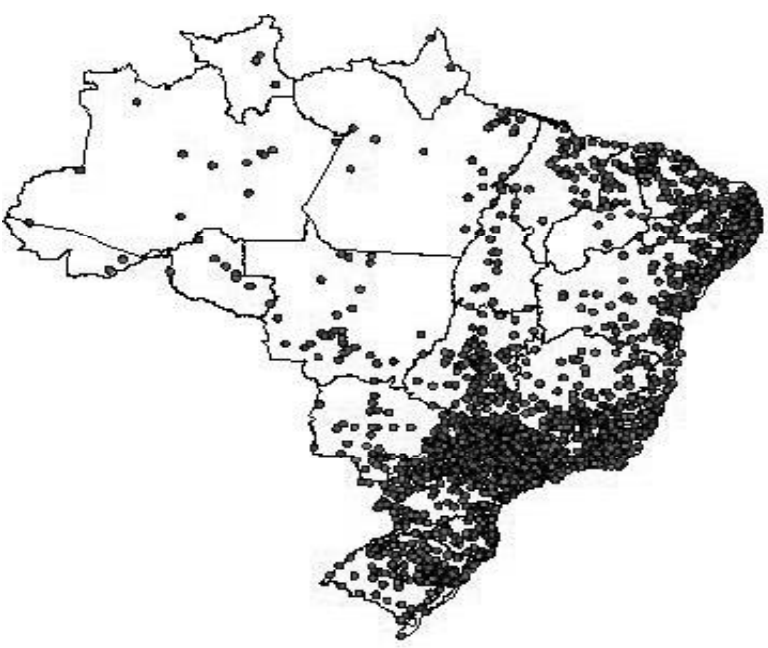

Figura 2 - Distribuição espacial dos municípios com pelo menos um caso de AIDS registrado, no Brasil, no período de 1987 a 1993 - Fonte: CN-DST/AIDS/SPS - Ministério da Saúde, 2000. 


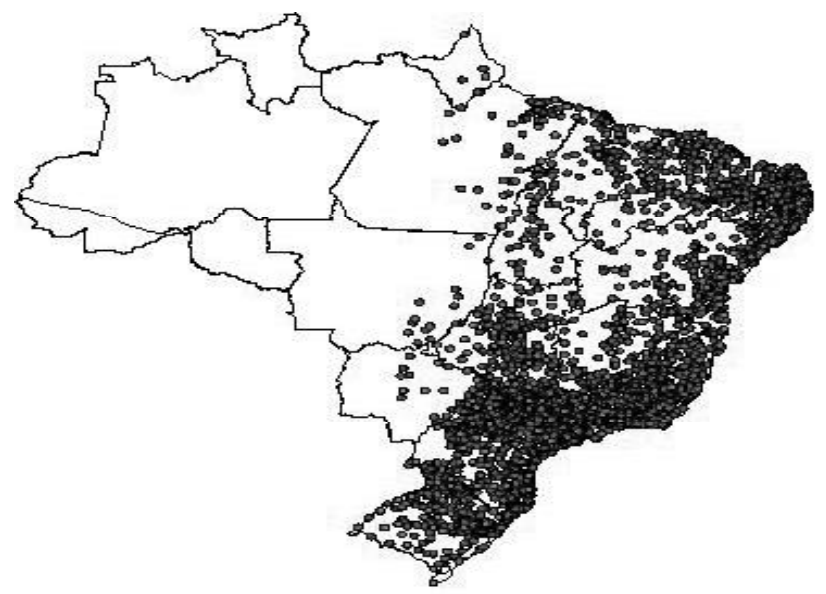

Figura 3 - Distribuição espacial dos municípios com pelo menos um caso de AIDS registrado, no Brasil, no período de 1984 a
1996 - Fonte: CN-DST/AIDS/SPS - Ministério da Saúde, 2000.

os dados de indivíduos com 15 anos ou mais de idade, a velocidade de crescimento da epidemia foi de aproximadamente $36 \%$ ao ano, no período de 1987-1989 a 1990-1992, decrescendo para 12\%, de 1990-1992 para 1993-1996. Constatou-se que, apesar de registrar as maiores taxas de incidência, a região Sudeste apresenta atualmente o menor ritmo de crescimento e a maior tendência à estabilidade. A análise da difusão da AIDS, segundo tamanho populacional dos municípios, mostra que a epidemia teve início nos grandes centros urbanos, mas esses detêm, atualmente, o menor aumento relativo.

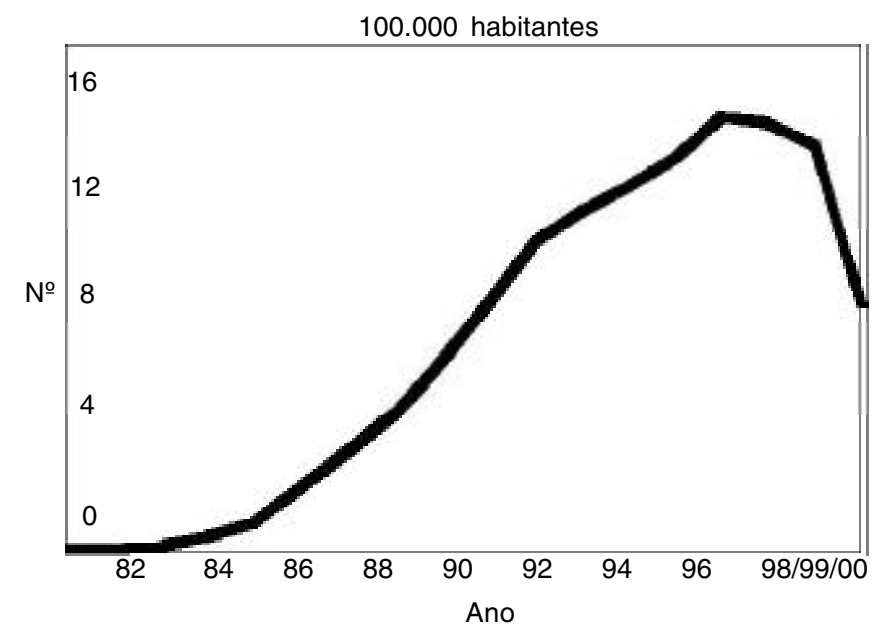

Figura 4 - Taxa de incidência de AIDS (por 100.000 habitantes) segundo ano de diagnóstico,Brasil, $1982-1999$ - Fonte: CN-DSTI AIDS/SPS - Ministério da Saúde, 2000.

Verificou-se ainda que a dinâmica da disseminação espacial da epidemia tem sido maior, nos últimos anos, entre municípios pequenos, com menos de 50 mil habitantes (Tabela 1). Trata-se de municípios mais pobres e de menor renda per capita. Ao contrário do que se observa para pequenos municípios, entre as cidades grandes as que apresentam mais de 500 mil habitantes, há desaceleração da velocidade de crescimento, com exceção da Região Sul. Essa região apresentou no período 1993-1996, a taxa de incidência mais elevada do País, superior a encontrada no Sudeste.

Tabela 1 - Taxas médias de incidência de AIDS (100.000 habitantes) por categoria populacional (município segundo número de habitantes) e período de tempo, Brasil, 1987-1996.

\begin{tabular}{lccr}
\hline $\begin{array}{c}\text { Categoria populacional } \\
\text { ( } \mathrm{n} \text { - hab) }\end{array}$ & \multicolumn{3}{c}{ Período de tempo } \\
\cline { 2 - 4 } & $1987-1989$ & $1990-1992$ & $1993-1996$ \\
\hline $0-50.000$ & 0,56 & 1,87 & 13,03 \\
$50.001-200.000$ & 2,31 & 7,73 & 27,30 \\
$200.001-500.000$ & 6,45 & 17,17 & 32,40 \\
500.001 e mais & 10,93 & 24,70 & 17,56 \\
\hline Total & 4,68 & 11,74 & \\
\hline
\end{tabular}

Fonte: CN-DST/AIDS/SPS - Ministério da Saúde, 2000

\section{MUDANCCAS NAS CATEGORIAS DE TRANSMISSÃO: A HETEROSSEXUALIZAÇÃO E A FEMINIZAÇÃO}

No início da epidemia, o segmento populacional constituído dos homens que fazem sexo com outros homens - homossexuais e bissexuais - foi o mais atingido. No ano de $1984,71 \%$ dos casos notificados eram referentes a homossexuais e bissexuais masculinos. Entretanto, à extensa disseminação inicial, seguiu-se certa estabilização em anos posteriores, em especial entre aqueles homens pertencentes aos estratos sociais médios urbanos, em todas as regiões do País, em meio aos quais verificou-se relevante mobilização social e mudança de comportamento no 
sentido de práticas sexuais mais seguras ${ }^{13}$, traduzindose em redução da participação desta subcategoria de exposição entre os casos notificados, correspondendo atualmente, $1999 / 2000$, a $16 \%$ dos casos.

Presentemente, no Brasil, a via de transmissão heterossexual constitui a mais importante característica da dinâmica da epidemia, com expressão relevante em todas as regiões. Houve incremento importante desta forma de transmissão: de 6,6 \% em 1988, para 39,2\%, em 1998 (Figura 5). Esta característica tem contribuído de modo decisivo para o aumento de casos em mulheres, traduzido na progressiva redução da razão de

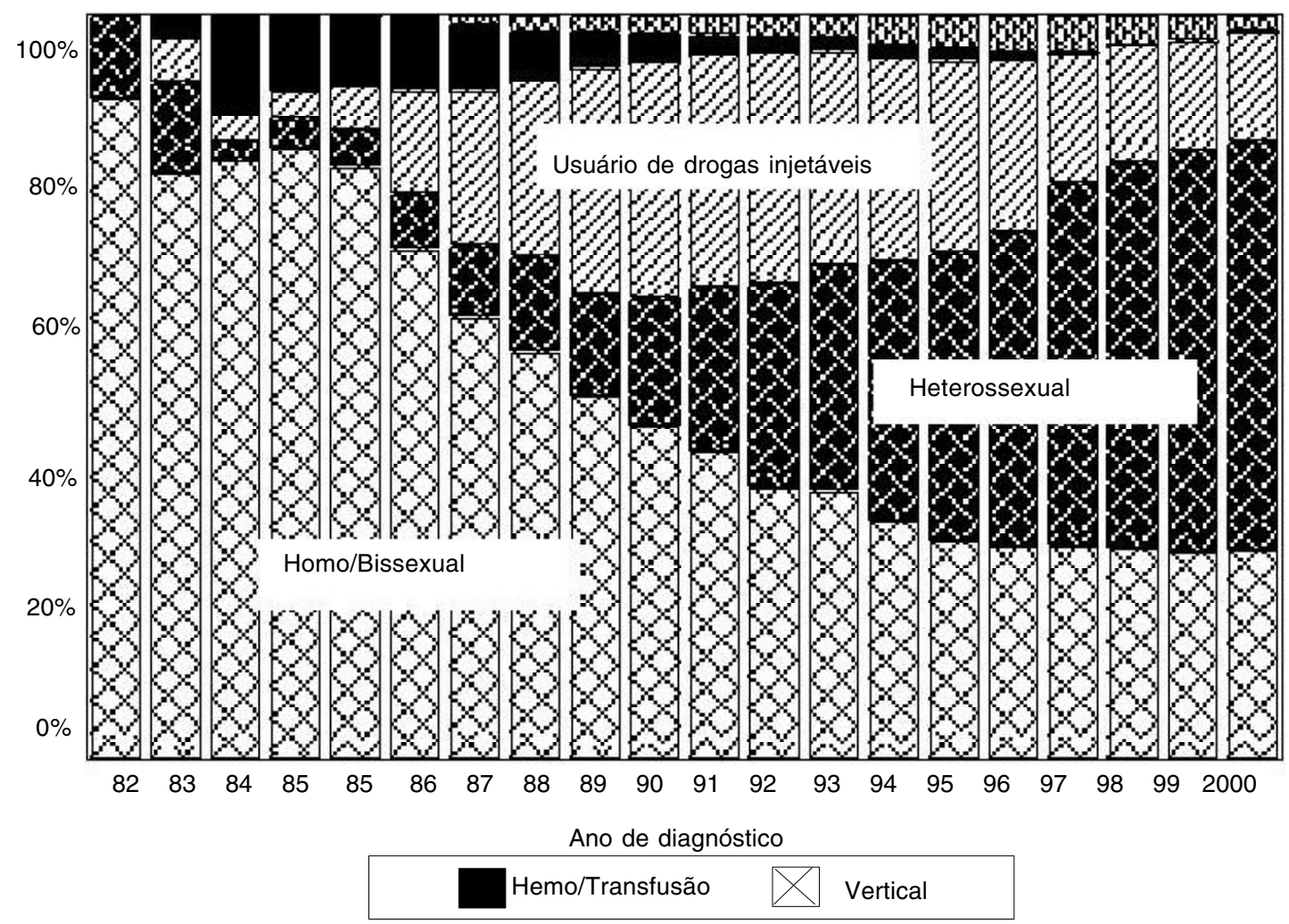

Figura 5 - Percentual de casos de AIDS por ano de diagnóstico e subcategoria de exposição. Brasil, 1982-2000*.

*Dados preliminares até 26/02/2000. Excluídos casos com categorias de exposição ignorada

sexo (dada pela razão entre os casos do sexo masculino e os casos do sexo feminino), no tempo e em todas as categorias de exposição. Os valores da razão de sexo passaram de 24:1, em 1985, para 6:1 em 1990, situandose em 2:1, desde 1997 (Figura 6). Curiosamente, as menores razões de sexo ocorrem nos municípios brasileiros com menos de 50 mil habitantes ${ }^{16}$. Aproximadamente $15 \%$ dos municípios que notificaram a doença já inverteram a relação de incidência entre os sexos. A relação dos casos de AIDS entre homens e mulheres que, desde 1997, vem se mantendo na ordem de 2 para 1 , já se inverteu em 229 dos 1.552 municípios que notificaram pelo menos 1 caso de AIDS no período de 1999/2000. A maioria desses 229 municípios (70\%) apresenta população menor ou igual a 50 mil habitantes, e a transmissão da doença nesses locais revelou-se predominantemente por contato heterossexual. Entre as mulheres, 57\% são donas de casa, em todos os níveis de escolaridade, na faixa etária de 20 a 39 anos. Essas ocorrências representam $5 \%$ dos casos registrados até junho de 2000.

Enquanto os maiores aumentos relativos dos casos em mulheres são observados na totalidade do território brasileiro, observa-se aparente desaceleração do crescimento da epidemia entre os homens da região Sudeste, particularmente nas grandes cidades, com taxa de variação de $4 \%$ ao ano, a menor do Brasil. O mesmo não se observa com as mulheres, cuja taxa de incidência nos municípios com mais de 500 mil habitantes, na própria região Sudeste, apresentou crescimento da ordem de $20 \%$ ao $a^{16}{ }^{16}$. Note que a proporção de casos masculinos notificados caiu de $90,1 \%$, de 1980 a 1988, para 66,6\%, em 1999/2000, evidenciando-se redução de $25 \%$, enquanto entre as 


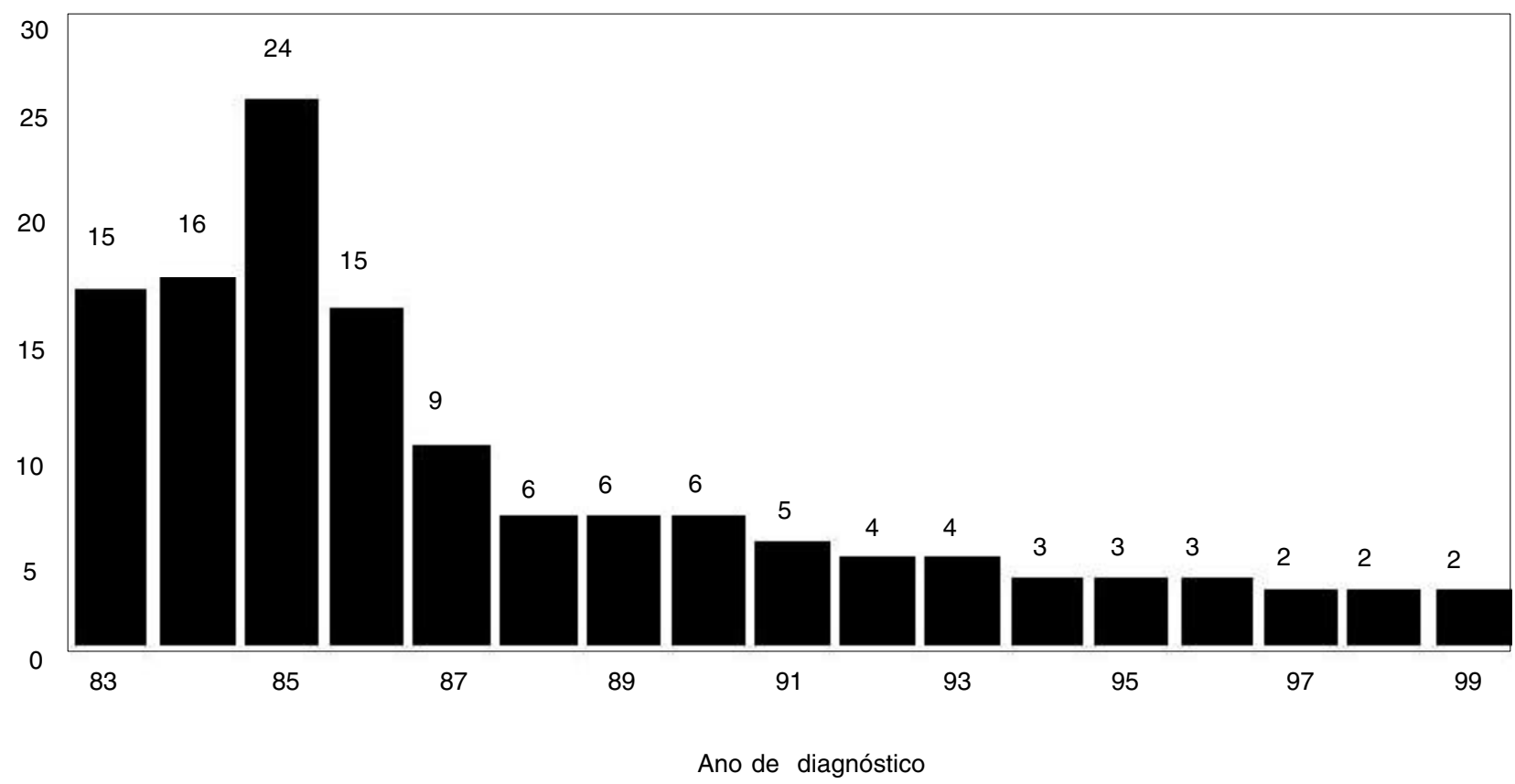

Figura 6 - Casos de AIDS - Razão de sexo, Brasil, 1983 a 1999. Fonte: CN-DST/AIDS/SPS - Ministério da Saúde, 2000

mulheres, a proporção de casos elevou-se de $9,9 \%$ para $33,3 \%$, nos períodos citados.

Quanto à categoria de transmissão sangüínea, anotaram-se alterações relevantes, principalmente em hemofílicos e em indivíduos que receberam transfusão de sangue. Segmentos populacionais intensamente atingidos no início da epidemia apresentaram importante declínio ao longo do tempo. Essa queda explica-se pelo controle do sangue e hemoderivados, principalmente com a disponibilidade dos testes laboratoriais para detecção de anticorpos anti-HIV, a partir de 1986. Em 1984, essas subcategorias representavam $62 \%$ dos casos da categoria de exposição sangüínea e, em 1999/2000, representam apenas $0,9 \%$. Enquanto isso, o segmento de usuários de drogas injetáveis (UDI) que, desde meados dos anos 80 , passou a ocupar posição de destaque entre os casos por transmissão sangüínea, mantém-se em expansão em determinadas áreas geográficas. Em 1984, 37\% dos casos por transmissão sangüínea eram atribuídos ao compartilhamento de agulhas e seringas, entre UDI. Essa subcategoria já representa $99 \%$ dos casos de AIDS por transmissão sangüínea ${ }^{12}$. A expressiva participação dos UDI, entre as ocorrências de AIDS, desempenhou papel central no processo de expansão da epidemia para municípios de pequeno e médio porte, a partir de disseminação inicial ao longo da faixa que conecta o
Centro-Oeste ao interior paulista ${ }^{1} \mathrm{e}$, mais recentemente, ao litoral sul do País ${ }^{2}$, bem como tem contribuído para o aumento de casos entre as mulheres usuárias de drogas injetáveis ou aquelas que contraíram o HIV por meio de relações sexuais com parceiros UDI.

No que pese a grande proporção de casos para os quais a categoria de exposição permanece ignorada, chegando a atingir $32 \%$ na região Centro-Oeste, tem-se constatado que as distribuições dos casos de AIDS por categoria de exposição nas grandes regiões demonstram padrões regionais distintos. Há predomínio da transmissão sexual para ambos os sexos, com baixas proporções de casos registrados em UDI, nas regiões Norte e Nordeste. De modo diverso, no Sul e no Sudeste, a categoria UDI possui papel destacado, com percentuais semelhantes de casos heterossexuais e UDI. Analisandose a evolução temporal das distribuições dos casos por categoria de exposição, pode-se verificar que as grandes cidades revelam padrão diferenciado. Nessas cidades, a categoria de homo/bissexuais prevalece em todos os períodos de tempo. No entanto, esta categoria tem sua relevância reduzida ao longo do tempo, proporcionalmente, à medida que cresce o número de casos por transmissão heterossexual. Quando se considera os municípios com populações de 50 a 200 mil habitantes e de 200 a 500 mil habitantes, evidencia-se a preponderância da categoria dos usuários de drogas injetáveis. Já nos municípios de menos 
de 50 mil habitantes, é nítido o aumento de casos por transmissão heterossexual ${ }^{16}$.

Com referência à idade, desde o começo da epidemia o grupo etário mais atingido, em ambos os sexos, tem sido o de 20 a 39 anos que, sistematicamente, representa mais de $60 \%$ dos casos de AIDS, perfazendo cerca de $70 \%$ do total de casos notificados até junho de 2000. Contudo, segundo Guimarães ${ }^{9}$, ao se analisar a proporção de casos com 13 a 29 anos de idade, observase que caiu de $37,3 \%$, de 1980 a 1988 , para $30,1 \%$, em 1998/1999; já os casos com 30 anos ou mais aumentou de $60,6 \%$ para $69,7 \%$ no mesmo período. Castilho et al ${ }^{5}$ destacam que o grupo homo/bissexuais masculinos é mais idoso (coortes das décadas de 40 e 50), enquanto os usuários de drogas injetáveis e casos de transmissão heterossexual, tanto em mulheres quanto em homens, encontram-se entre os mais jovens (coortes das décadas de 60 e 70). Outro destaque para a descrição dos casos quanto à idade, refere-se aos menores de 13 anos: a predominância da transmissão perinatal nos casos pediátricos modificou o perfil etário da ocorrência dos mesmos. Até o ano de 1987, 21\% das crianças notificadas com AIDS, tinham de 0 a 4 anos, enquanto em $1999 / 2000$, cerca de $86 \%$ dos casos pediátricos encontravam-se nesse grupo etário.

Com relação à transmissão vertical do HIV, ou seja, a transmissão da mãe infectada para o seu concepto durante a gravidez, parto ou aleitamento natural, houve aumento progressivo dessa categoria ao decorrer do tempo, como conseqüência direta da maior participação feminina entre os casos de AIDS no Brasil. Em 1986, esta categoria correspondia a $0,2 \%$ do total de casos e, em 1999/2000, representa 2,6\%. Desde o registro da primeira ocorrência de transmissão perinatal, em 1985 , até 03/06/2000, notificaram-se 5.409 casos nessa forma de transmissão, o que representa cerca de $2,8 \%$ do total acumulado de casos $^{12}$.

\section{PERFIL SÓCIO-ECONÔMICO DOS CASOS DE AIDS: A PAUPERIZAÇÃO}

Ainda que com restrições, utiliza-se a escolaridade como variável proxis de situação sócioeconômica e o fenômeno de pauperização tem sido caracterizado pelo aumento da proporção de casos de AIDS em indivíduos com baixa escolaridade. Houve expressiva mudança no perfil da escolaridade dos casos notificados entre adultos e adolescentes. A totalidade dos casos de AIDS, com escolaridade conhecida, diagnosticados até 1982, apresentava nível superior ou médio; já em 1985, o percentual deste grupo alcançou $76 \%$, enquanto apenas $24 \%$ dos casos eram analfabetos ou cursaram os primeiros quatro anos do ensino fundamental. Nos anos subseqüentes, houve tendência progressiva de aumento no registro de casos em indivíduos com menor grau de escolaridade, observando-se em 1999/2000, entre os casos com escolaridade informada que, $74 \%$ eram analfabetos ou haviam completado o ensino fundamental, e apenas
$26 \%$ apresentavam mais de 11 anos de escolaridade ou curso superior.

Fonseca et $\mathrm{al}^{7}$, analisando a evolução temporal dos casos de AIDS por grau de escolaridade, para indivíduos com 20 a 69 anos de idade, de 1989 a 1996, verificaram que a incidência dos casos com baixa escolaridade (menos de 8 anos de escola, incluindo os analfabetos) vem apresentando tendência nitidamente crescente para o sexo masculino, atingindo níveis próximos de $70 \%$, no ano de 1996 . Para as mulheres, desde o início da série temporal estudada, as proporções no grupo de menor escolaridade já ultrapassavam $70 \%$ e permaneceram estáveis. Todavia, para aqueles indivíduos com mais de 8 anos de escolaridade, observou-se redução dos casos no sexo masculino, o mesmo não ocorrendo no sexo feminino (Tabela 2). Segundo Castilho et $\mathrm{al}^{5}$ há, também, diferença significativa nas ocorrências em analfabetos segundo o sexo: $2,8 \%$ dos homens são analfabetos, sendo de $5,1 \%$ a cifra entre mulheres.

$\mathrm{O}$ estudo de Fonseca et $\mathrm{al}^{7}$ revela ainda diferenças inter-regionais quanto à escolaridade, embora essas diferenças não tenham se mostrado tão marcantes como as encontradas por sexo. Destaca-se as elevadas taxas de variação anual observadas nas regiões Sul (30\%) e Centro-Oeste (28\%) entre os casos masculinos com baixo grau de escolaridade (1ำ $\mathrm{grau})$. A região Sul foi a única a registrar aumento relativo significativo entre os casos de $2^{\circ}$ grau. Para o sexo feminino, as diferenças regionais tornam-se evidentes. De forma semelhante ao observado para os casos masculinos, a região Sul apresenta as maiores taxas de variação para os dois grupos de escolaridade. Nas regiões Norte, Nordeste e Centro-Oeste, a taxa de variação anual revelou-se maior entre as mulheres com menor nível de instrução.

Em adultos usuários de drogas injetáveis verificase que, independentemente do sexo, há considerável predominância de indivíduos com baixa escolaridade desde o início da epidemia. Essa mesma situação, embora em menor escala, observa-se quando se considera os heterossexuais, entre os quais também predomina a ocorrência de casos com baixa ou nenhuma escolaridade.

\section{MODIFICAC̄̃̃ES NA HISTÓRIA NATURAL DA AIDS: A REDUÇÃO DA MORBI-MORTALIDADE}

Além das tendências de interiorização, heterossexualização, feminização e pauperização, já expostos anteriormente, nota-se crescente aumento da sobrevida dos casos de AIDS no Brasil. Os avanços tecnológicos e o melhor conhecimento da etiopatogenia da AIDS permitiram o surgimento de novas propostas de intervenções diagnósticas, profiláticas e terapêuticas, às quais pode ser atribuído o expressivo aumento da sobrevida dos doentes ${ }^{4}$. Além do que, a evolução dos conhecimentos e da utilização de tecnologias cada vez 
Tabela 2 - Taxas de incidência de AIDS, segundo sexo e grau de escolaridade* para indivíduos com 20-69 anos de idade por ano de diagnóstico, Brasil, 1990-97

\begin{tabular}{|c|c|c|c|c|c|c|c|c|c|c|c|}
\hline \multirow[t]{2}{*}{ Sexo } & \multirow{2}{*}{$\begin{array}{l}\text { Escolaridade } \\
\text { (anos) }\end{array}$} & \multicolumn{8}{|c|}{ Ano Diagnóstico } & \multirow{2}{*}{$\begin{array}{l}\text { Taxa de } \\
\text { variação } \\
\text { anual }(\%)^{\text {** }}\end{array}$} & \multirow{2}{*}{$p^{* \star *}$} \\
\hline & & 90 & 91 & 92 & 93 & 94 & 95 & 96 & 97 & & \\
\hline \multirow[t]{2}{*}{ Masculino } & até 8 & 14,7 & 19,9 & 25,2 & 28,3 & 30,4 & 31,7 & 33,4 & 35,0 & 17,6 & 0,001 \\
\hline & + de 8 & 31,3 & 36,9 & 41,7 & 41,2 & 40,8 & 39,6 & 37,0 & 34,3 & 4,9 & 0,056 \\
\hline \multirow[t]{2}{*}{ Feminino } & até 8 & 3,1 & 4,7 & 6,8 & 8,6 & 9,6 & 11,0 & 13,3 & 15,6 & 25,7 & 0,000 \\
\hline & + de 8 & 2,2 & 3,1 & 5,1 & 6,0 & 6,7 & 7 & 8,6 & 9,5 & 24,5 & 0,001 \\
\hline \multirow[t]{2}{*}{ Total } & até 8 & 8,8 & 12,2 & 15,9 & 18,3 & 19,8 & 21 & 23,2 & 25,1 & 16,3 & 0,003 \\
\hline & + de 8 & 16,1 & 19,2 & 22,5 & 22,7 & 22,9 & 22,9 & 22,1 & 21,3 & 4,8 & 0,047 \\
\hline
\end{tabular}

* Casos com escolaridade ignorada foram corrigidos por um modelo de análise discriminante. ${ }^{* \star}$ As tendências foram analisadas para o período de 1990 -1996. ${ }^{* \star}$ Valores do nível de significância descritivo

Fonte: CN-DST/AIDS/SPS - Ministério da Saúde, 2000

mais eficazes fez com que a AIDS tivesse sua história natural alterada. Com desfecho letal rápido nos primeiros casos descritos, a doença assumiu caráter crônico, tendo atualmente evolução prolongada, onde longos períodos assintomáticos são interrompidos pelo surgimento de infecções oportunistas.

Há indicações de que o tratamento anti-retroviral combinado possa também alterar a história natural de doenças e condições clínico-imunológicas associadas à AIDSS. No Brasil, apesar da disponibilidade de medicamentos anti-retrovirais mais eficazes desde 1995-1996, incluindo inibidores da protease, não há estudos de prospecção de indivíduos infectados pelo HIV com o objetivo de definir o comportamento das condições clínicas associadas. No que pese as limitações metodológicas quanto à confiabilidade dos dados, o atraso na notificação, bem como os critérios de notificação e diagnóstico, estudo realizado com base em registro de casos notificados ao Ministério da Saúde, adultos, de 1980 a maio de 1999, considerando as oito principais condições associadas clinicamente mais específicas $^{9}$, revelou queda estatisticamente significativa de quase todas as doenças oportunistas. Comparandose o período inicial (1980-1988) e final (1998-1999), houve redução da incidência de candidíase de $62,3 \%$ para $43,8 \%$; de $25,8 \%$ a $17,9 \%$ para a tuberculose; de $27,7 \%$ a $11,9 \%$ para a pneumonia por Pneumocystis carinii; de $14,4 \%$ a $10 \%$ para neurotoxoplasmose; de $14,9 \%$ a $9,1 \%$ para o herpes simples; de $11,4 \%$ a $2,1 \%$ para o sarcoma de Kaposi; de 6,6\% a 2,6\% para a criptococose e de 6,2\% a 2,2\% para as infecções intestinais por protozoários. Apesar da redução global de incidência das condições associadas, o estudo revelou que houve aumento da incidência de tuberculose e neurotoxoplasmose para as regiões Nordeste e Centro-Oeste. Constatou, também, que a tuberculose apresentou maior incidência entre os doentes com baixa escolaridade (até a oitava série do ensino fundamental), enquanto a pneumonia por Pneumocystis carinii e o sarcoma de Kaposi tiveram maiores incidências entre aqueles com maior escolaridade (ensino médio e superior), apesar de declínios semelhantes.

A queda dos números absolutos de óbitos por AIDS nos municípios do Rio de Janeiro e São Paulo (Figuras 7 e 8), no período de 1995 a 1999, aponta para a redução da letalidade sendo, em ambos municípios, mais acentuada entre os casos do sexo masculino ${ }^{6}$.

Apesar dos progressos alcançados na redução da mortalidade, o impacto da AIDS ainda mantém-se particularmente importante na mortalidade em adultos em idade produtiva, tendo sido a $4^{\mathrm{a}}$ causa de óbito no grupo de 20 a 49 anos de idade, em 1996, em ambos os sexos ${ }^{11}$. Esse impacto nessa faixa etária provoca, ainda, como conseqüência, grande número de órfãos, decorrentes da morte materna por AIDS, estimado em 29.928, entre 1987 e 1999, além dos problemas decorrentes dessa situação ${ }^{14}$.

\section{INFECÇÕES PELO HIV: ESTIMATIVA E PROJEÇÕES}

Os dados discutidos no itens anteriores referem-se a casos de AIDS - situação clínica que traduz progressiva perda da função do sistema imune resultante da infecção pelo HIV - que observam os critérios estabelecidos pelo Sistema Nacional de Vigilância Epidemiológica do Ministério da Saúde ${ }^{11}$. Considerando o longo período de latência entre a infecção pelo vírus e o aparecimento da AIDS, a análise com base nos casos notificados implica o fato de lidar com infecções ocorridas há cerca de cinco a dez anos. Esta base de dados, embora importante para o mapeamento epidemiológico da epidemia da doença, possibilitando comparações mundiais, apresenta limitações, sobretudo na vigência dos avanços da terapia anti-retroviral. A tendência, com as profilaxias e tratamentos atuais, é de que as pessoas infectadas pelo HIV permaneçam 


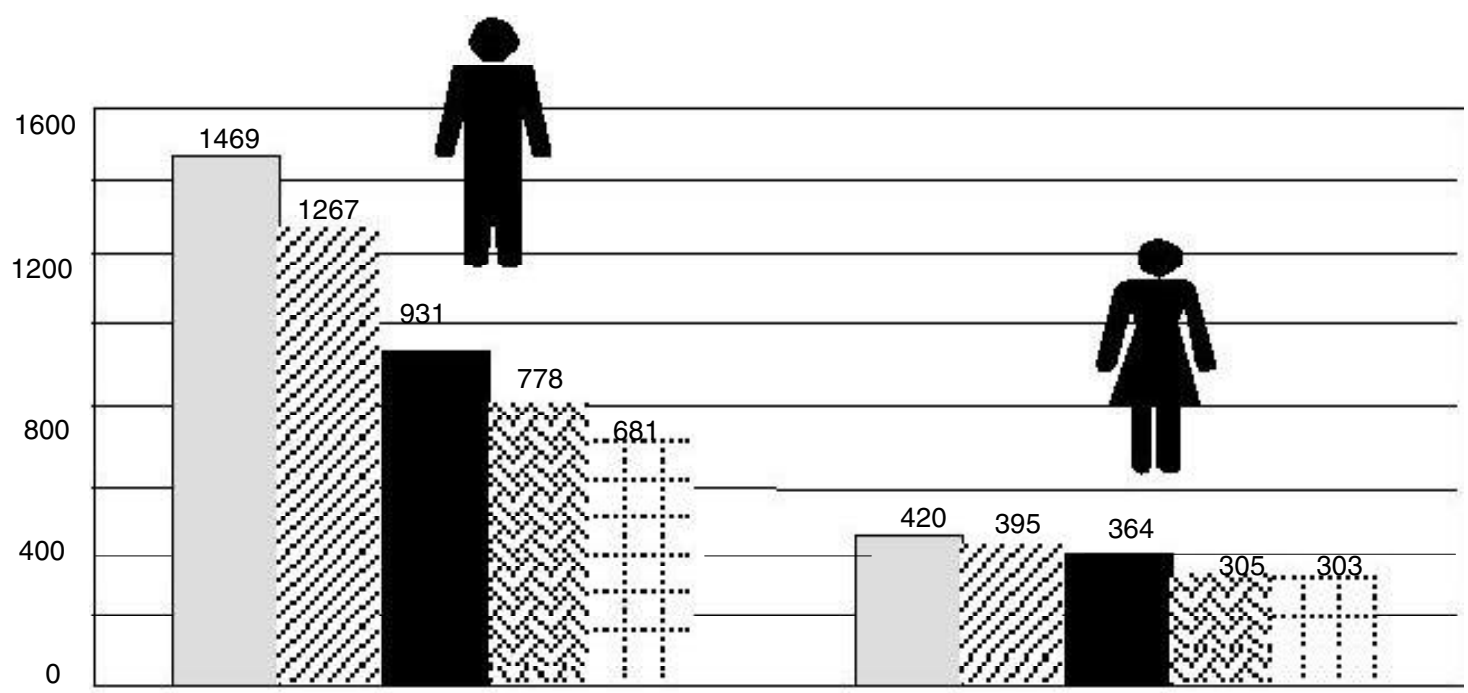

$\begin{array}{llllllll}1995 & 1996 & 0 & 1997 & 0 & 1998 & : & 1999\end{array}$

Figura 7 - Óbitos por AIDS em números absolutos, segundo sexo. Município de São Paulo - 1995 - 1999. Fonte: CN-DST/AIDS/SPS - Ministério da Saúde, 2000

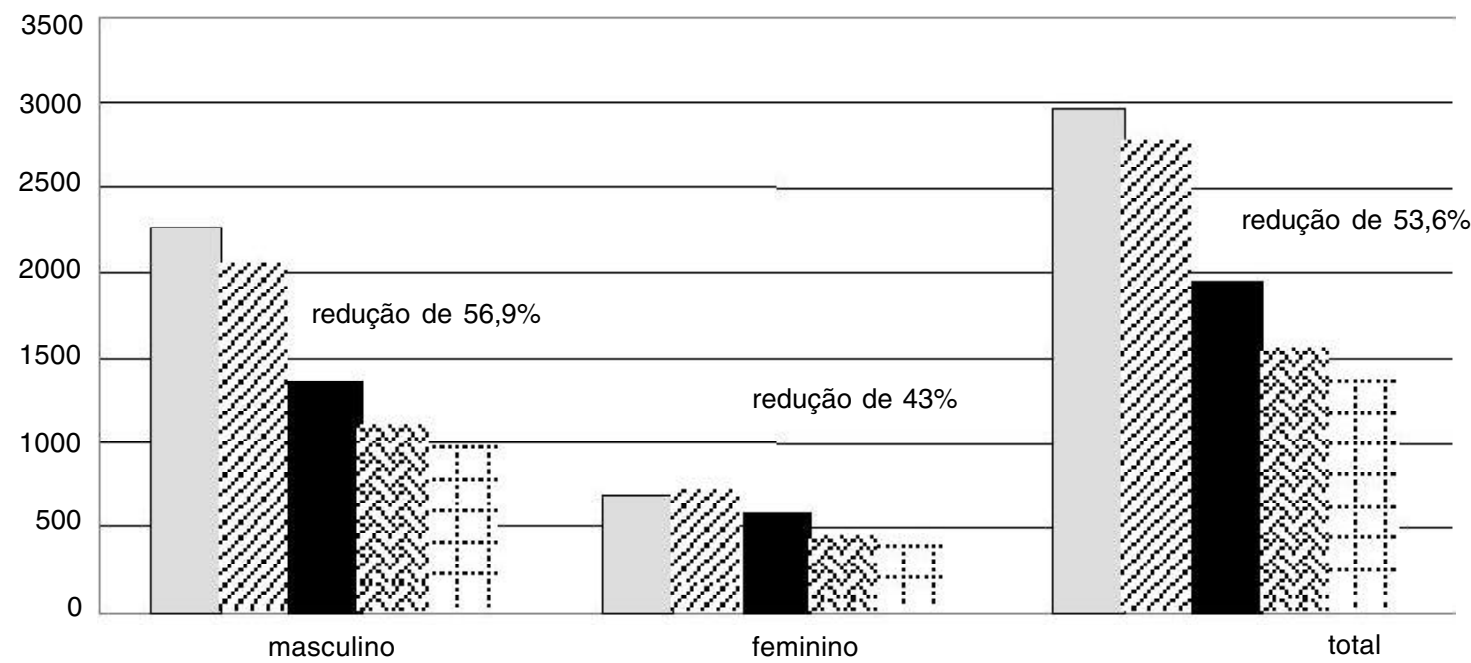

\section{5 $1996 \square$ 1997[}

Figura 8 - Óbitos por AIDS em números absolutos, segundo sexo. Município do Rio de Janeiro - 1995 - 1999. Fonte: CN-DST/AIDS/SPS - Ministério da Saúde, 2000

indefinidamente nesta condição, sem necessariamente preencherem os critérios de definição de caso de AIDS e, portanto, sem serem notificados. Essa situação acarreta distorções na interpretação da evolução da epidemia, com sérias conseqüências para atividades de planejamento das ações de prevenção e assistência. Portanto, torna- se necessário distinguir, quando da análise da epidemia do HIV e da AIDS, os estudos sobre AIDS daqueles referentes à infecção pelo HIV. Essa última situação é de maior magnitude e, geralmente, compreende indivíduos assintomáticos.

O conhecimento da epidemia da infecção pelo HIV fundamenta-se em estimativas. Essas estimativas são 
estabelecidas, principalmente, pela aplicação de modelos matemáticos baseados no número de casos notificados de AIDS, nos resultados de estudos de soroprevalência em populações-sentinelas, e em inquéritos sorológicos pontuais em populações específicas, de baixo e alto risco para a exposição ao HIV.

Szwarcwald e Castilho ${ }^{14}$ estimaram em 536.920 o número de pessoas de 15-49 anos e o de 12.898 o número de gestantes infectadas pelo HIV no Brasil em 1998. Os autores tomaram como base os dados dos estudos de população-sentinela em gestantes no País e a razão de crescimento da taxa de incidência acumulada de AIDS em mulheres de 15-34 anos, do período de 1990-1992 para 1993-1996. Os resultados das estimativas do número de infectados, segundo sexo e para as cinco grandes regiões brasileiras encontram-se contemplados na Figura 9. Veja na Figura 10 as estimativas das proporções de gestantes infectadas pelo HIV segundo macrorregião.

O número estimado de 12.898 corresponde a $0,4 \%$ do total das gestantes e, segundo dados do Ministério da Saúde, há registro de que apenas 2.512 receberam zidovudina injetável no momento do parto, o que corresponde à modesta cifra de $19,5 \%$ das gestantes infectadas. Considerando a disponibilidade contínua da zidovudina injetável nos serviços públicos de saúde e ao fato de que a maioria das gestantes aceita se submeter ao diagnóstico sorológico da infecção pelo HIV e ao tratamento com zidovudina, não seria de se esperar tão pequeno percentual recebendo o medicamento injetável durante o parto. Essa realidade motivou a organização do Sistema de Vigilância de Gestantes HIV Positivas e Crianças Expostas, atualmente em fase de implantação no Brasil, que permitirá, desde o rastreamento da infecção no pré-natal - condição inicial para o desencadeamento das ações profiláticas -, até a mensuração da prevalência da infecção infantil, produto final e indicador de impacto das ações profiláticas desenvolvidas. Além disso, o acompanhamento contínuo da prevalência da infecção entre as gestantes deve fornecer indicador mais representativo da infecção entre as mulheres ${ }^{6}$.

Estudos comportamentais, em curso, procuram identificar fatores preditores da dinâmica da infecção pelo HIV. Um desses estudos, a Pesquisa do Comportamento Sexual da População Brasileira e Percepção do HIV/AIDS ${ }^{3}$, conduzido em 183 microrregiões urbanas do Brasil, em amostra de 3.600 indivíduos de 16 a 65 anos no ano de 1998, revelou que $80 \%$ das pessoas nessa faixa etária são sexualmente ativas, sendo os homens mais ativos sexualmente ( $87 \%$ ) do que as mulheres $(72 \%)$. A grande maioria da população sexualmente ativa ( $89 \%)$ situa-se na faixa etária de 20 a 40 anos. Do total da população sexualmente ativa, $81 \%$ referiram relações estáveis nos

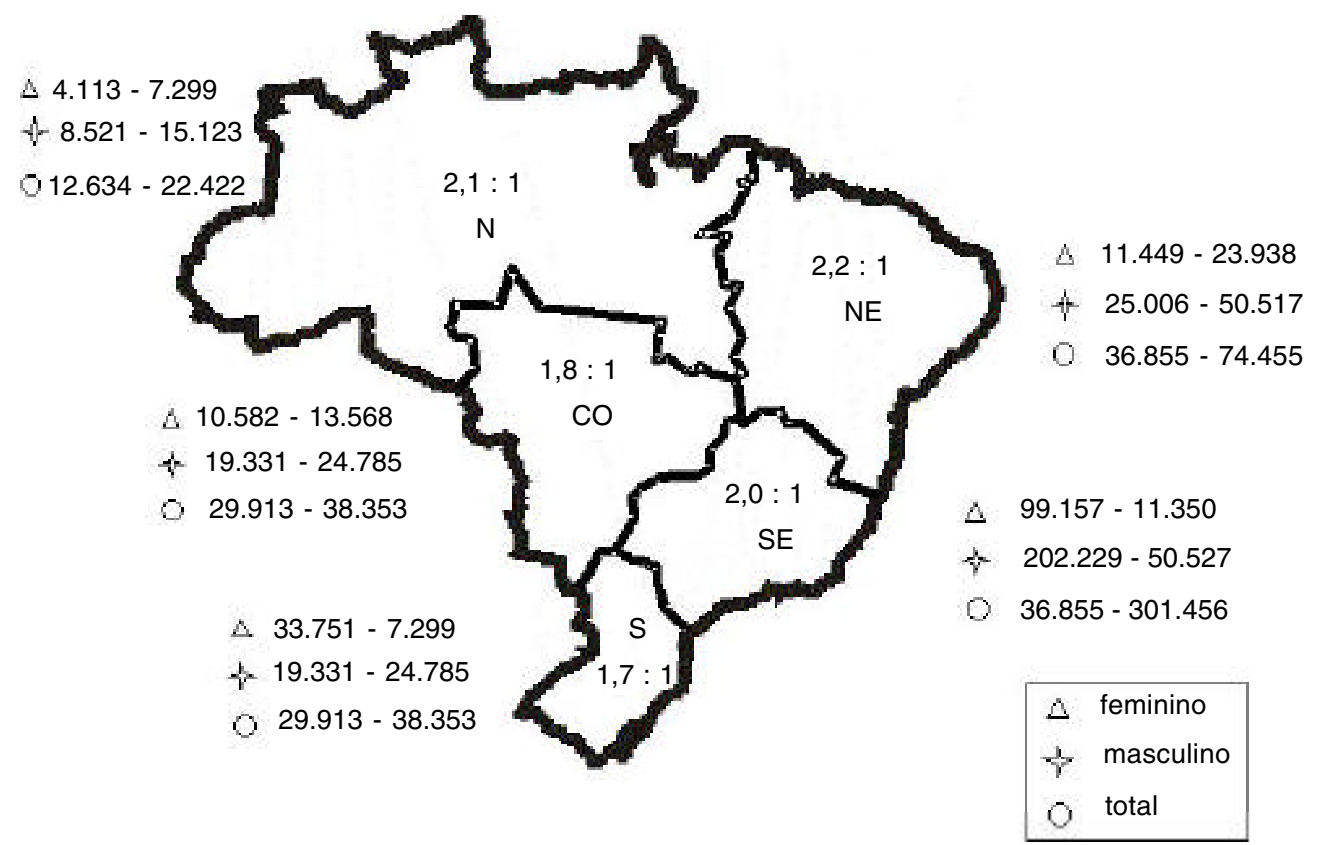

Figura 9 - Estimativa do número de infectados pelo HIV na faixa etária de 15 a 49 anos por macrorregião, Brasil 1998*. Fonte: CN-DST/AIDS/SPS - Ministério da Saúde, 2000

*Baseado no intervalo de 68\% para proporções de infecção pelo HIV entre gestantes de 15 a 34 anos 


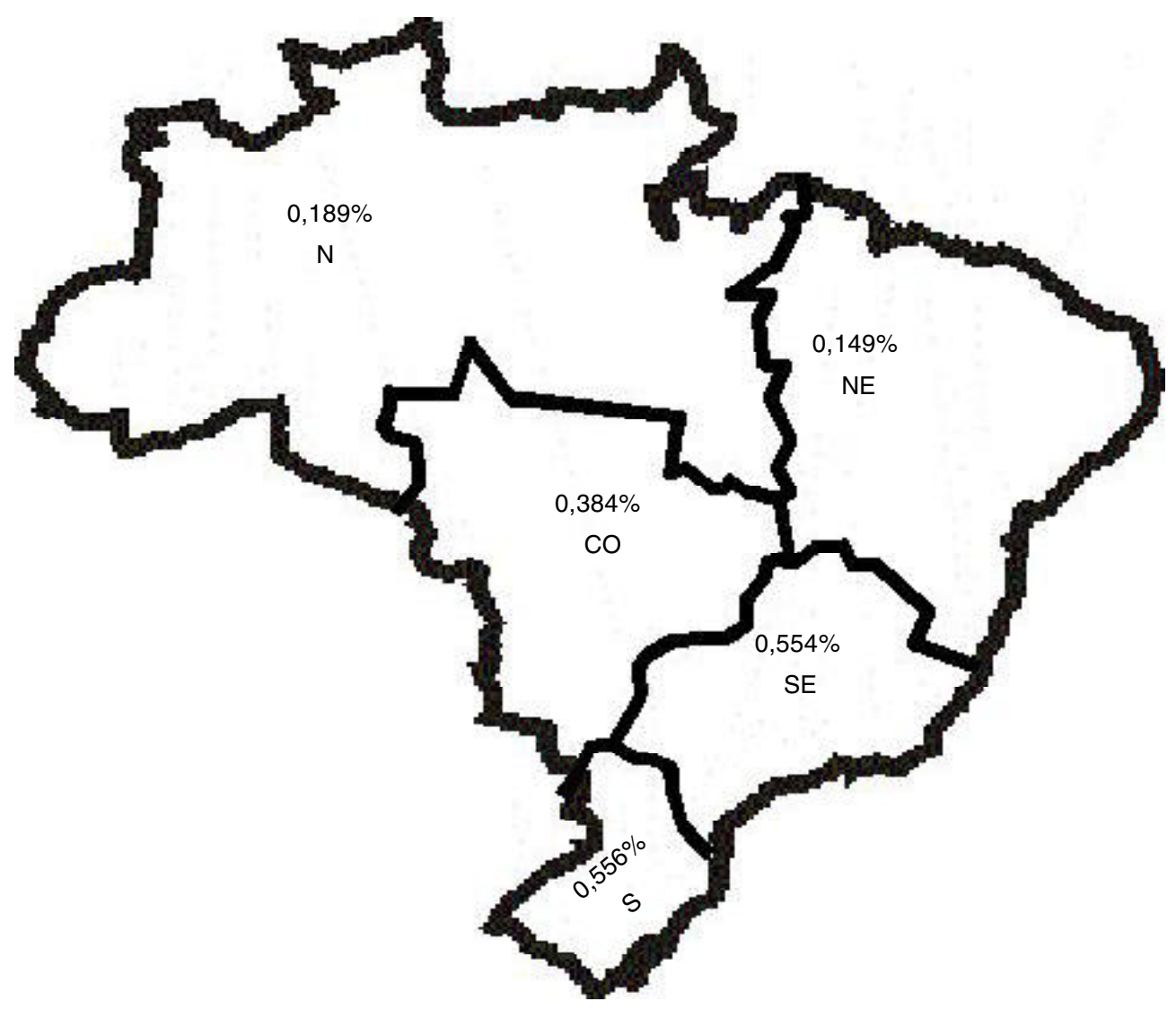

Figura 10 - Estimativa da proporção de gestantes infectadas pelo HIV segundo macrorregião, Brasil, 1998. Fonte: CN-DST/AIDS/SPS - Ministério da Saúde, 2000

últimos 12 meses, 6\% informaram relações eventuais e $13 \%$ mantinham os dois tipos de relações. No mesmo período de tempo, $71 \%$ das mulheres relataram relações sexuais com um único parceiro, contra $46 \%$ dos homens. Aproximadamente, $35 \%$ das pessoas entrevistadas relataram relações sexuais com dois ou mais parceiros e $6 \%$ tiveram mais de cinco parceiros no período.

O mesmo estudo demonstrou que grande parte das pessoas já se sentiu exposta à infecção pelo HIV. Cerca de $20 \%$ desses submeteram-se a exames sorológicos para detectar infecção pelo HIV em serviços públicos ou privados, estando a maioria na faixa etária de 26 a 40 anos. Ao se analisar o uso do preservativo, $64 \%$ da população sexualmente ativa referiram ter usado ou estar usando o preservativo, nos últimos 12 meses. Entre os mais jovens, esse percentual atinge $87 \%$. Quarenta e oito por cento dos que disseram estarem usando ou terem usado o preservativo, o fizeram pela primeira vez nos últimos cinco anos, demonstrando adesão crescente na utilização desse método de prevenção. Este achado guarda coerência com dados da venda de preservativos que demonstram crescimento em anos mais recentes, passando de 50 milhões de unidades no ano de 1992, para 300 milhões de unidades em 1998.

A Pesquisa Comportamental de Conscritos do Exército Brasileiro, realizada por ocasião da apresentação de jovens de 17 a 20 anos, no ano de 1998, em amostra de 30.318 indivíduos em todo o País ${ }^{17}$ revelou a vulnerabilidade dos adolescentes às infecções sexualmente transmissíveis . Na análise dos resultados do estudo, selecionaram-se três estratos: o primeiro formado por municípios de cinco estados do Norte e Centro-Oeste (estrato N/CO); o segundo formado por municípios dos estados da região Sul (estrato S); e o terceiro formado por municípios dos Estados do Rio de Janeiro e São Paulo (estrato RJ/SP). O estudo baseou-se em questionário preenchido pelos conscritos no momento de sua apresentação ao Exército e de coleta de sangue para teste de infecção pelo HIV. Obtiveramse informações a respeito das condições sócioeconômicas, práticas sexuais, uso de drogas injetáveis e problemas relacionados às infecções sexualmente transmissíveis, permitindo associá-las aos resultados dos exames sorológicos para o HIV. 
Vale a pena ressaltar que os conscritos residentes no Norte e Centro-Oeste apresentaram maior taxa de atividade sexual, idade mais precoce de início do relacionamento sexual, maior número de parceiros no último ano, menor taxa de uso de preservativos e maior percentual de uso de drogas injetáveis. Também observou-se nesse estrato a maior proporção de infectados pelo HIV $(0,2 \%)$, sendo de $0,08 \%$ no RJ/SP e de $0,12 \%$ no Sul. A pesquisa apontou ainda que alta proporção de jovens usou preservativos na última relação sexual. Ao se analisar o uso do preservativo nos últimos 12 meses, observouse que a sua utilização diminuiu quando o parceiro era fixo, aumentando progressivamente se o parceiro é casual, pagante ou pago.

Outro achado relevante diz respeito à escolaridade como a variável de maior poder explicativo para as diferenças relativas às práticas sexuais de risco. A freqüência do uso de preservativo aumenta de acordo com o grau de escolaridade, enquanto o uso de drogas diminui com o aumento da escolaridade. Esses resultados corroboram os estudos realizados em outros países em que jovens e adolescentes de baixo nível de instrução e baixo nível sócio-econômico são mais susceptíveis às infecções sexualmente transmissíveis.

\section{AGRADECIMENTOS} Figuras.

Os autores agradecem à Coordenação de DST e AIDS do Ministério da Saúde pela cessão das Tabelas e

\section{REFERÊNCIAS BIBLIOGRÁFICAS}

1. Barcellos C, Bastos FI. Redes sociais e difusão da AIDS no Brasil. Boletín de la Oficina Sanitaria Panamericana 121:1144,1996.

2. Bastos FI, Strathdee AS, Derrico M, Pina F. Drug use and the spread of HIV/AIDS in South America and the Caribbean. Drugs: Education, Prevention \& Policy 6:29-50, 1999.

3. Berquó E (coord). Comportamento sexual da população brasileira e percepções do HIV/AIDS. Ministério da Saúde, Série Avaliação, nำ 4, Brasília, 2000.

4. Brito AM. A epidemia de AIDS em Pernambuco: sobrevida dos doentes no período de 1983 a 1995. Tese de Mestrado. Universidade Federal de Pernambuco, Recife, PE, 1997.

5. Castilho EA, Chequer P, Szwarcwald CL. A AIDS no Brasil. In: Rouquayrol E, Almeida N (eds) Epidemiologia, Saúde. Editora Médica e Científica, Rio de Janeiro, 1999.

6. Dhalia C, Barreira D, Castilho EA. A AIDS no Brasil: situação atual e tendências. Boletim Epidemiológico - AIDS XIII (1): 3-13, SE 48/99 a 22/00, 2000.

7. Fonseca MG, Bastos FI, Derrico M, Andrade CLT, Travassos C, Szwarcwald CL. AIDS e grau de escolaridade no Brasil: evolução temporal de 1986 a 1996. Cadernos de Saúde Pública, Rio de Janeiro 16 (supl 1):77-87, 2000.

8. Fonseca MGP, Castilho EA. Os casos de AIDS entre usuários de drogas injetáveis. Brasil, 1980-1997. Boletim Epidemiológico - AIDS, X (2): 6-14, SE 23 a 35, 1997.

9. Guimarães MDC. Estudo temporal das doenças associadas à AIDS no Brasil. Cadernos de Saúde Pública, Rio de Janeiro 16 (supl 1): 21-36, 2000.
10. Mann J, Tarantola DJM, Netter TW (coord). A AIDS no mundo. ABIA da Universidade Estadual do Rio de Janeiro, Editora Relume Dumará, Rio de Janeiro,1993.

11. Ministério da Saúde. Boletim Epidemiológico - AIDS XII (2):5-16, SE 48/98 a 08/99, 1999.

12. Ministério da Saúde. Boletim Epidemiológico - AIDS XIII (1): 15-56, SE 48/99 a 22/00, 2000.

13. Parker R. A construção da solidariedade: AIDS, sexualidade e política no Brasil. ABIA da Universidade Estadual do Rio de Janeiro, Editora Relume Dumará, Rio de Janeiro,1994.

14. Szwarcwald CL, Andrade CLT, Castilho EA. Estimativa do número de órfãos decorrentes da AIDS materna, Brasil, 1987-1999. Cadernos de Saúde Pública, Rio de Janeiro 16 (supl 1):129-134, 2000.

15 Szwarcwald CL, Bastos FI, Esteves MAP, Andrade CLT. A disseminação da epidemia da AIDS no Brasil, no período de 19871996: uma análise espacial. Cadernos de Saúde Pública, Rio de Janeiro 16 (supl 1):7-19, 2000.

16. Szwarcwald CL, Castilho EA. Estimativa do número de pessoas de 15 a 49 anos infectadas pelo HIV, Brasil, 1998. Cadernos de Saúde Pública, Rio de Janeiro 16 (supl 1):135-141, 2000

17. Szwarcwald CL, Castilho EA, Barbosa-Jr A, Gomes MRO, Costa EAMM, Maletta BV, Carvalho RFM, Oliveira SR, Chequer P. Comportamento de risco dos conscritos do Exército Brasileiro, 1998: uma apreciação da infecção pelo HIV segundo diferenciais sócio-econômicos. Cadernos de Saúde Pública, Rio de Janeiro 16 (supl 1):113-128, 2000.

18. World Bank. Confronting AIDS: Public Priorities in a Global Epidemic. Oxford University Press, Washington,1997. 